\title{
EVEN DEGREE CHARACTERS IN PRINCIPAL BLOCKS
}

\author{
EUGENIO GIANNELLI, GUNTER MALLE, AND CAROLINA VALLEJO RODRÍGUEZ
}

\begin{abstract}
We characterise finite groups such that for an odd prime $p$ all the irreducible characters in its principal $p$-block have odd degree. We show that this situation does not occur in non-abelian simple groups of order divisible by $p$ unless $p=7$ and the group is $M_{22}$. As a consequence we deduce that if $p \neq 7$ or if $M_{22}$ is not a composition factor of a group $G$, then the condition above is equivalent to $G / \mathbf{O}_{p^{\prime}}(G)$ having odd order.
\end{abstract}

\section{INTRODUCTION}

Let $G$ be a finite group, let $p$ be a prime dividing the order of $G$ and let $B_{0}$ be the Brauer principal ( $p$-)block of $G$. Brauer's height zero conjecture asserts that $p$ does not divide the degrees of the irreducible ordinary characters belonging to $B_{0}$ if, and only if, a Sylow $p$-subgroup $P$ of $G$ is abelian. Let $q$ be a prime different from $p$. It would be interesting to characterise when all degrees of irreducible ordinary characters belonging to $B_{0}$ are coprime with $q$. When $q=2, \mathrm{G}$. Navarro predicted that all irreducible ordinary characters in $B_{0}$ have odd degree if, and only if, $G / \mathbf{O}_{p^{\prime}}(G)$ has odd order. We confirm here that this claim holds whenever $p \neq 7$. For $p=7$, the group $M_{22}$ is a counterexample, and the only counterexample among finite simple groups.

Theorem A. Let $p$ be an odd prime, and let $B_{0}$ be the principal p-block of a group $G$ of order divisible by $p$. If $p \neq 7$ or $M_{22}$ is not a composition factor of $G$, then every irreducible character in $B_{0}$ has odd degree if, and only if, $G / \mathbf{O}_{p^{\prime}}(G)$ is a group of odd order.

It is worth mentioning that G. Navarro, G. Robinson and P.H. Tiep [NRT17 have recently characterised the groups for which $G / \mathbf{O}_{p^{\prime}}(G)$ has odd order in terms of the number of real-valued characters in the principal $p$-block of $G$. Namely they have shown that the principal $p$-block $B_{0}$ contains only one real-valued irreducible character if, and only if, $G / \mathbf{O}_{p^{\prime}}(G)$ has odd order.

The proof of Theorem A ultimately relies on the fact that for every finite non-abelian simple group $G$ and every odd prime divisor $p$ of its order, the principal $p$-block of $G$ contains an even degree character, except for $p=7$ and $G=M_{22}$.

2010 Mathematics Subject Classification. 20C15, 20C20, 20C30, $20 \mathrm{C} 33$.

Key words and phrases. Principal blocks, even degree characters.

The first author's research is funded by Trinity Hall, University of Cambridge. The second author gratefully acknowledges financial support by SFB TRR 195. The third author is partially supported by the Spanish Ministerio de Educación y Ciencia Proyectos MTM2016-76196-P and the ICMAT Severo Ochoa project SEV-2011-0087. 
Theorem B. Let $G$ be a non-abelian simple group of order divisible by an odd prime $p$. Let $B_{0}$ be the principal p-block of $G$. Then $B_{0}$ contains an irreducible character $\chi$ of even degree unless $G=M_{22}$ and $p=7$.

In order to show that Theorem B holds for alternating groups we prove a more general result that we believe to be of independent interest, concerning the principal block of symmetric (and alternating) groups.

Theorem C. Let $n \geq 5$, and let $p$ and $q$ be distinct primes with $q<p \leq n$. Let $B_{0}$ be the principal p-block of either $\mathfrak{S}_{n}$ or $\mathfrak{A}_{n}$. Then $B_{0}$ contains an irreducible character $\chi$ of degree divisible by $q$.

This article is structured as follows. In Section 2 we prove that Theorem A holds assuming Theorem B. The rest of the article is devoted to the proof of Theorem B on finite non-abelian simple groups. In Section 3 we prove Theorem C, which includes the alternating group case of Theorem B. Finally, in Section 4 we show that Theorem B holds for sporadic groups and for groups of Lie type, and conclude using the Classification of Finite Simple Groups.

We follow the notation of [Is76] for ordinary characters and the notation of [Nav98] for blocks. By a block, we shall mean a $p$-block. Also, if $B$ is a block of $G$, we will denote by $\operatorname{Irr}(B)$ the set of irreducible complex characters lying in the block $B$. In general, we will denote by $B_{0}(H)$ the principal block of a group $H$. Finally if $n$ is a natural number, then we denote by $\nu_{p}(n)$ the maximal integer $k$ such that $p^{k}$ divides $n$.

Acknowledgement. Part of this work was done while the third author was visiting the Department of Mathematics at the TU Kaiserslautern. She would like to thank everyone at the department for the warm hospitality. The authors would also like to thank Gabriel Navarro for his insight.

\section{A Reduction to Finite Simple Groups}

In this section we assume that Theorem B holds and we prove Theorem A. In this sense, we reduce Theorem A to a problem on finite simple groups.

We assume that the reader is familiar with the theory of blocks and normal subgroups (see for example Chapter 9 of [Nav98]). Also, we recall that if $G_{1}$ and $G_{2}$ are finite groups, then $\operatorname{Irr}\left(B_{0}\left(G_{1} \times G_{2}\right)\right)=\operatorname{Irr}\left(B_{0}\left(G_{1}\right)\right) \times \operatorname{Irr}\left(B_{0}\left(G_{2}\right)\right)$ (this follows directly from the definition of the principal block, see [Nav98, Def. 3.1]).

Proof of Theorem $A$. We remark that $\operatorname{Irr}\left(B_{0}\right) \subseteq \operatorname{Irr}\left(G / \mathbf{O}_{p^{\prime}}(G)\right)$, since $B_{0}$ covers the principal block of $\mathbf{O}_{p^{\prime}}(G)$ which only consists of the trivial character. Also, whenever $N \unlhd G$, we have that $\operatorname{Irr}\left(B_{0}(G / N)\right) \subseteq \operatorname{Irr}\left(B_{0}\right)$.

The "if part" follows from the first remark. To prove the "only if part" we first show that the hypotheses imply the $p$-solvability of $G$. Otherwise, since the hypotheses are inherited by quotients of $G$, we may assume that there is a minimal normal subgroup $N$ of $G$ which is neither a $p$-group nor a $p^{\prime}$-group. Hence $N=\prod_{i=1}^{t} S^{x_{i}}$, where $S \unlhd N$ is a simple non-abelian group of order divisible by $p$ and $x_{i} \in G$. By hypothesis, either $p \neq 7$ or $S \neq M_{22}$. By Theorem B, let $\theta \in \operatorname{Irr}\left(B_{0}(S)\right)$ have even degree. Then $\phi=\theta^{x_{1}} \times \cdots \times \theta^{x_{t}}$ 
has even degree and belongs to $\operatorname{Irr}\left(B_{0}(N)\right)=\prod_{i=1}^{t} \operatorname{Irr}\left(B_{0}(S)\right)^{x_{i}}$. By [Nav98, Thm. 9.4], some $\chi \in \operatorname{Irr}\left(B_{0}\right)$ lies over $\phi$. Since $\chi(1)$ is odd by hypothesis, we get a contradiction.

As $G$ is $p$-solvable, we have that $\operatorname{Irr}\left(B_{0}\right)=\operatorname{Irr}\left(G / \mathbf{O}_{p^{\prime}}(G)\right)$, by [Nav98, Thm. 10.20]. Hence, the hypothesis that all irreducible characters in $B_{0}$ have odd degree implies that the group $G / \mathbf{O}_{p^{\prime}}(G)$ has a normal Sylow 2-subgroup by the Ito-Michler theorem (see [It51] and [Mi86]). This forces $G / \mathbf{O}_{p^{\prime}}(G)$ to be a group of odd order, as desired.

Recall that our motivation is to characterise when a prime $q$ does not divide the degrees of the irreducible characters in the principal block. The Ito-Michler theorem characterises when a prime $q$ does not divide the degrees of the irreducible characters of a group. A natural version of the Ito-Michler theorem for principal blocks would be: If all the irreducible characters of $B_{0}(G)$ have degree coprime to $q$, for some prime $q \neq p$, then some Sylow $q$-subgroup $Q$ of $G$ is normalised by a Sylow $p$-subgroup $P$ of $G$. In [NW01] the authors prove this result under the assumption that $G$ is a $\{p, q\}$-separable group. However, such a version does not hold outside $\{p, q\}$-separable groups, as the authors also point out that the separability condition of $G$ is necessary (as shown by $G=J_{1}, p=2$, $q=5)$. For $q=2$ and $p \neq 7$ we have the characterisation given by Theorem A.

\section{Alternating Groups}

The aim of this section is to prove Theorem C, which in particular implies Theorem B for alternating groups.

We start by recalling some facts in the representation theory of symmetric groups. We refer the reader to [Ja79], [JK81] or [O194] for a more detailed account. A partition $\lambda=\left(\lambda_{1}, \lambda_{2}, \ldots, \lambda_{\ell}\right)$ is a finite non-increasing sequence of positive integers. We say that $\lambda$ is a partition of $|\lambda|=\sum \lambda_{i}$, written $\lambda \vdash|\lambda|$. The Young diagram of $\lambda$ is the set $[\lambda]=\left\{(i, j) \in \mathbb{N} \times \mathbb{N} \mid 1 \leq i \leq \ell, 1 \leq j \leq \lambda_{i}\right\}$, where we orient $\mathbb{N} \times \mathbb{N}$ with the $x$-axis pointing right and the $y$-axis pointing down. We denote by $\lambda^{\prime}$ the conjugate partition of $\lambda$, whose Young diagram is obtained from the Young diagram of $\lambda$ by a reflection over the main diagonal.

Given $(r, c) \in[\lambda]$, the corresponding hook $H_{(r, c)}(\lambda)$ is the set defined by

$$
H_{(r, c)}(\lambda)=\{(r, y) \in[\lambda] \mid y \geq c\} \cup\{(x, c) \in[\lambda] \mid x \geq r\} .
$$

We set $h_{r, c}(\lambda)=\left|H_{(r, c)}(\lambda)\right|=1+\left(\lambda_{r}-c\right)+\left(\lambda_{c}^{\prime}-r\right)$, and we denote by $\mathcal{H}(\lambda)$ the multiset of hook-lengths in $[\lambda]$. Similarly we let $\mathcal{H}_{r}(\lambda)$ be the multiset of hook-lengths in the $r$ th row of $[\lambda]$. For $e \in \mathbb{N}$ we let $\mathcal{H}^{e}(\lambda)=\bigcup_{r=1}^{\ell} \mathcal{H}_{r}^{e}(\lambda)$, where $\mathcal{H}_{r}^{e}(\lambda)=\left\{(r, c) \in[\lambda] \mid e\right.$ divides $\left.h_{r, c}(\lambda)\right\}$. If $(r, c) \in \mathcal{H}^{e}(\lambda)$, then we say that $H_{(r, c)}(\lambda)$ is an $e$-hook of $\lambda$, so that $\left|\mathcal{H}^{e}(\lambda)\right|$ is the number of $e$-hooks of $\lambda$. The $e$-core $C_{e}(\lambda)$ of $\lambda$ is the partition obtained from $\lambda$ by successively removing all $e$-hooks.

Let $q$ be a prime number. An important combinatorial object for our analysis is the $q$-core tower $T^{q}(\lambda)$ of a partition $\lambda$ of $n$ (we refer the reader to [O194, Chap. II] for a comprehensive description of this object). Every partition of a given natural number is uniquely determined by its $q$-core tower. We write $T(\lambda)$ instead of $T^{q}(\lambda)$ when $q$ is clear from the context. For $j \in \mathbb{N}_{0}$ we denote by $T_{j}(\lambda)$ the $j$ th layer of $T(\lambda)$. As explained in 
full details in O194, $T_{j}(\lambda)=\left(\mu_{1}, \ldots, \mu_{q^{j}}\right)$ is a sequence of $q$-core partitions such that

$$
\sum_{j \geq 0}\left|T_{j}(\lambda)\right| q^{j}=n, \quad \text { where } \quad\left|T_{j}(\lambda)\right|=\left|\mu_{1}\right|+\cdots+\left|\mu_{q^{j}}\right| .
$$

Note that $\left|T_{0}(\lambda)\right|=\left|C_{q}(\lambda)\right|$. Also note that if $n=\sum_{j=0}^{k} \alpha_{j} q^{j}$ is the $q$-adic expansion of $n$, then $\left|T_{s}(\lambda)\right|=0$ whenever $s>k$. It is useful to remark at this point that if $m$ is the maximal integer such that $\left|T_{m}(\lambda)\right| \neq 0$, then we have that $\left|\mathcal{H}^{q^{m}}(\lambda)\right|=\left|T_{m}(\lambda)\right|$.

Partitions of $n$ correspond canonically to the irreducible characters of $\mathfrak{S}_{n}$. We denote by $\chi^{\lambda}$ the irreducible character naturally labelled by $\lambda \vdash n$. We recall that $\left(\chi^{\lambda}\right)_{\mathfrak{A}_{n}}$ is irreducible if, and only if, $\lambda \neq \lambda^{\prime}$. Otherwise $\left(\chi^{\lambda}\right)_{\mathfrak{A}_{n}}=\phi+\phi^{g}$ for some $\phi \in \operatorname{Irr}\left(\mathfrak{A}_{n}\right)$ and $g \in \mathfrak{S}_{n} \backslash \mathfrak{A}_{n}$. (See [JK81, Thm. 2.5.7].)

The following result was first proved by MacDonald [Mac71] and it is crucial for our purposes.

Theorem 3.1 (MacDonald). Let $q$ be a prime and let $n$ be a natural number with q-adic expansion $n=\sum_{j=0}^{k} \alpha_{j} q^{j}$. Let $\lambda$ be a partition of $n$. Then

$$
\nu_{q}\left(\chi^{\lambda}(1)\right)=\left(\sum_{j \geq 0}\left|T_{j}(\lambda)\right|-\sum_{j=0}^{k} \alpha_{j}\right) /(q-1) .
$$

Useful consequences of this result are recorded in the following two lemmas.

Lemma 3.2. Let $q, n$ and $\lambda$ be as in Theorem [3.1. If $q$ does not divide $\chi^{\lambda}(1)$ then $\left|\mathcal{H}^{q^{k}}(\lambda)\right|=\alpha_{k}$.

Lemma 3.3. Let $k \geq 2$ and let $n=2^{k+1}-2^{\ell}$, for some $\ell \leq k-2$. Let $\lambda \vdash n$ such that $\left|\mathcal{H}^{2^{k}}(\lambda)\right|=0$ and $\left|\mathcal{H}^{2^{k}-1}(\lambda)\right| \leq 2$. Then $\nu_{2}\left(\chi^{\lambda}(1)\right) \geq 2$.

Proof. Since $\left|\mathcal{H}^{2^{k}}(\lambda)\right|=0$ we deduce that $\left|T_{k}(\lambda)\right|=0$. Hence we have that $\left|T_{k-1}(\lambda)\right|=$ $\left|\mathcal{H}^{2^{k-1}}(\lambda)\right| \leq 2$. We know that $n=\sum_{j=0}^{k}\left|T_{j}(\lambda)\right| 2^{j}$, hence we obtain that $\sum_{j=0}^{k}\left|T_{j}(\lambda)\right| \geq$ $k+3-\ell$. Since $n$ has $k+1-\ell$ binary digits, we deduce that $\nu_{2}\left(\chi^{\lambda}(1)\right) \geq 2$ from Theorem 3.1 .

Finally, we state the following consequence of Nakayama's Conjecture (proved independently by R. Brauer and G. de B. Robinson, see e.g. [Ja79] or [O194]). The second statement follows from [Nav98, Thm. 9.2].

Proposition 3.4. Let $p$ be a prime and let $n=a+p w$ for some $a \in\{0,1, \ldots, p-1\}$ and $w \in \mathbb{N}$. Let $\lambda \vdash n$. Then $\chi^{\lambda}$ lies in the principal p-block of $\mathfrak{S}_{n}$ if, and only if, $C_{p}(\lambda)=(a)$. Moreover every irreducible constituent of $\left(\chi^{\lambda}\right)_{\mathfrak{A}_{n}}$ lies in the principal p-block of $\mathfrak{A}_{n}$.

In order to prove Theorem C, we devote most of our efforts to show that the following slightly stronger statement holds.

Theorem 3.5. Let $q$ and $p$ be primes and let $n \geq 5$ be a natural number such that $q<p \leq n$. Then there exists an irreducible character $\chi$ in the principal p-block of $\mathfrak{S}_{n}$ such that $q$ divides $\chi(1)$. Moreover, if $\phi$ is an irreducible constituent of the restriction $\chi_{\mathfrak{A}_{n}}$, then $q$ divides $\phi(1)$. 
We split the proof of Theorem 3.5 into a series of lemmas (dealing with symmetric groups) and corresponding corollaries (concerning alternating groups). We fix below the notation that will be kept for the rest of this section.

Notation 3.6. Let $2 \leq q<p$ be prime numbers and let $n \in \mathbb{N}$, which we write as $n=a+p w$ for uniquely determined $a \in\{0,1, \ldots, p-1\}$ and $w \in \mathbb{N}$. Moreover let

$$
n=\alpha_{k} q^{k}+\sum_{j=0}^{k-1} \alpha_{j} q^{j}
$$

be the $q$-adic expansion of $n$, where $\alpha_{k} \neq 0$. We need also to fix the notation for the $q$-adic expansions of $p w$ and $a$. We let

$$
a=\beta_{k} q^{k}+\sum_{j=0}^{k-1} \beta_{j} q^{j} \quad \text { and } \quad p w=\zeta_{k} q^{k}+\sum_{j=0}^{k-1} \zeta_{j} q^{j},
$$

where $\beta_{j}, \zeta_{j} \in\{0,1, \ldots, q-1\}$ for all $j \in\{0, \ldots, k-1\}$ and $0 \leq \beta_{k} \leq \zeta_{k} \leq \alpha_{k} \leq q-1$. Note that $p w \neq \zeta_{k} q^{k}$, as both $\zeta_{k}$ and $q$ are smaller than $p$.

Finally we denote by $\mathcal{B}_{n}(q, p)$ the set consisting of all partitions $\lambda$ of $n$ such that $\chi^{\lambda}$ lies in the principal $p$-block of $\mathfrak{S}_{n}$ and such that $q$ divides $\chi^{\lambda}(1)$.

Lemma 3.7. Suppose that $a=0$. Then $\lambda=\left(\alpha_{k} q^{k}, 1^{n-\alpha_{k} q^{k}}\right) \in \mathcal{B}_{n}(q, p)$.

Proof. It is easy to see that $C_{p}(\lambda)$ is the empty partition. Therefore $\chi^{\lambda}$ lies in the principal block of $\mathfrak{S}_{n}$, by Proposition [3.4. The hook-length $h_{1,1}(\lambda)=w p$ is not divisible by $q^{k}$. Moreover, for all $j \in\left\{2,3, \ldots, \alpha_{k} q^{k}\right\}$ we have $h_{1, j}(\lambda)+(j-2)=h_{1,2}(\lambda)=\alpha_{k} q^{k}-1$. Hence we deduce that there are exactly $\alpha_{k}-1$ boxes $(1, d) \in[\lambda]$ such that $q^{k}$ divides $h_{1, d}(\lambda)$. It follows that $\left|\mathcal{H}_{1}^{q^{k}}(\lambda)\right|=\alpha_{k}-1$. Since $h_{2,1}(\lambda)=n-\alpha_{k} q^{k}<q^{k}$ we deduce that $\left|\mathcal{H}_{j}^{q^{k}}(\lambda)\right|=0$ for all $j \geq 2$. We conclude that $\left|\mathcal{H}^{q^{k}}(\lambda)\right|=\alpha_{k}-1$ and therefore that $q$ divides $\chi^{\lambda}(1)$, by Lemma 3.2 .

We have preferred to show in our proof of Lemma 3.7 the strategy that will be used to prove Lemmas 3.9 and 3.11 below, instead of using the well-known fact that whenever $\mu=\left(n-d, 1^{d}\right)$ is a hook partition the degree $\chi^{\mu}(1)$ is equal to the binomial coefficient $\left(\begin{array}{c}n-1 \\ d\end{array}\right)$. In any case, the necessary analysis of the divisibility of the binomial coefficient would not have sensibly shortened our argument.

Corollary 3.8. Let $a$ and $\lambda$ be as in Lemma 3.7 and let $\phi$ be an irreducible constituent of $\left(\chi^{\lambda}\right)_{\mathfrak{A}_{n}}$. Then $\phi$ lies in the principal p-block of $\mathfrak{A}_{n}$ and $q$ divides $\phi(1)$.

Proof. Since $\phi(1) \in\left\{\chi^{\lambda}(1), \chi^{\lambda}(1) / 2\right\}$ the statement follows trivially from Lemma 3.7, when $q \neq 2$. Similarly if $q=2$ and $\lambda \neq \lambda^{\prime}$ then, $\phi=\left(\chi^{\lambda}\right)_{\mathfrak{A}_{n}}$; and hence the statement follows, again by Lemma 3.7 .

Suppose that $\lambda=\left(2^{k}, 1^{n-2^{k}}\right)=\lambda^{\prime}$. Then $n=2^{k+1}-1$ and we observe that $\left|\mathcal{H}^{2^{k}}(\lambda)\right|=0$ and that $\left|\mathcal{H}^{2^{k-1}}(\lambda)\right|=2$. Using Lemma 3.3 we deduce that $\nu_{2}\left(\chi^{\lambda}(1)\right) \geq 2$ and therefore that $\phi(1)=\chi^{\lambda}(1) / 2$ is even.

From now on, we assume that $1 \leq a \leq p-1$. 
Lemma 3.9. Suppose that $w p>\alpha_{k} q^{k}$ and let $\lambda=\left(\alpha_{k} q^{k}-1, a+1,1^{n-\left(\alpha_{k} q^{k}+a\right)}\right)$. Then $\lambda \in \mathcal{B}_{n}(q, p)$ for all $n \neq\left(\alpha_{k}+1\right) q^{k}-1$. For $n=\left(\alpha_{k}+1\right) q^{k}-1$, we construct $\mu \in \mathcal{B}_{n}(q, p)$ as follows:

$$
\mu= \begin{cases}\left(a, 2,1^{p-2}\right) & \text { if } q=2, w=1 \quad \text { and } a=p-3 \\ \left(\alpha_{k} q^{k}-2, a+1,1^{n-\left(\alpha_{k} q^{k}+a-1\right)}\right) & \text { otherwise. }\end{cases}
$$

Proof. Let $n \neq\left(\alpha_{k}+1\right) q^{k}-1$. Note that $h_{1,1}(\lambda)=n-a=w p$. This implies that $C_{p}(\lambda)=(a)$. Moreover, $h_{1,1}(\lambda)$ is not divisible by $q^{k}$. Analysing other hook-lengths in $[\lambda]$, we see that for all $j \in\left\{3, \ldots, \lambda_{1}\right\}$ we have $1 \leq h_{1, j}(\lambda)<h_{1,2}(\lambda)=\alpha_{k} q^{k}-1$. It follows that $\left|\mathcal{H}_{1}^{q^{k}}(\lambda)\right| \leq \alpha_{k}-1$. On the other hand, $h_{2,1}(\lambda)=n-\left(\alpha_{k} q^{k}-1\right)<q^{k}$ and therefore $\left|\mathcal{H}_{j}^{q^{k}}(\lambda)\right|=0$ for all $j \geq 2$. We deduce that $\left|\mathcal{H}^{q^{k}}(\lambda)\right| \leq \alpha_{k}-1$ and hence that $q$ divides $\chi^{\lambda}(1)$, by Lemma 3.2. Notice that for $n=\left(\alpha_{k}+1\right) q^{k}-1$ we have that $h_{2,1}(\lambda)=q^{k}$. For this reason we need to construct a different partition in this case.

Let $\mu=\left(\alpha_{k} q^{k}-2, a+1,1^{n-\left(\alpha_{k} q^{k}+a-1\right)}\right)$. Then the composition $\mu$ defines a partition of $n$ unless $\mu_{2}>\mu_{1}$. This happens if, and only if, $a \geq \alpha_{k} q^{k}-2$ and in turn this is equivalent to the following chain of inequalities:

$$
n=a+p w \geq\left(\alpha_{k} q^{k}-2\right)+\left(\alpha_{k} q^{k}+1\right)=n+\left(\alpha_{k}-1\right) q^{k} \geq n .
$$

This is consistent if, and only if, we have equalities everywhere. Equivalently we must have $\alpha_{k}=1, p w=q^{k}+1$ and $a=q^{k}-2=p w-3$. Moreover, it is now easy to see that $p w-a=3$ forces $w=1$. Hence we have that $p=q^{k}+1$, which implies that $q=2$. The above discussion shows that $\mu_{2}>\mu_{1}$ if, and only if, $q=2, w=1$ and $a=p-3$. In this case we set $\mu=\left(a, 2,1^{p-2}\right)$. Observing that $h_{2,1}(\mu)=q^{k}+1$ and arguing exactly as before we verify that $\mu \in \mathcal{B}_{n}(q, p)$ (in both cases).

Corollary 3.10. Let $a, w p, \lambda$ and $\mu$ be as in Lemma 3.9. Let $\delta \in\{\lambda, \mu\}$ and let $\phi$ be an irreducible constituent of $\left(\chi^{\delta}\right)_{\mathfrak{A}_{n}}$. Then $\phi$ lies in the principal $p$-block of $\mathfrak{A}_{n}$ and $q$ divides $\phi(1)$.

Proof. Exactly as in the proof of Corollary [3.8, we can focus on the case where $q=2$. If $n=2^{k+1}-1$ then $\delta=\mu \neq \mu^{\prime}$ and therefore $\chi^{\mu}(1)=\phi(1)$ is even. If $n \neq 2^{k+1}-1$ then $\lambda=\lambda^{\prime}$ if and only if $a=1$ and $n=2^{k+1}-2$. Analysing the hook-lengths in $[\lambda]$ we observe that $\left|\mathcal{H}^{2^{k}}(\lambda)\right|=0$ and that $\left|\mathcal{H}^{2^{k-1}}(\lambda)\right| \leq 2$. Using Lemma 3.3 we deduce that $\nu_{2}\left(\chi^{\lambda}(1)\right) \geq 2$ and therefore that $\phi(1)=\chi^{\lambda}(1) / 2$ is even.

Lemma 3.11. Suppose that $w p<\alpha_{k} q^{k}$. If $\beta_{k}>0$, then define $\lambda \vdash n$ as follows:

$$
\lambda=\left(a, a-\beta_{k} q^{k}+1,1^{n-2 a+\beta_{k} q^{k}-1}\right) .
$$

Otherwise let $\lambda \vdash n$ be defined by:

$$
\lambda= \begin{cases}(n-2,2) & \text { if } a=1, \\ \left(a, 1^{w p}\right) & \text { if } a>1 \text { and } n \neq \alpha_{k} q^{k}, \\ \left(a, 2,1^{w p-2}\right) & \text { if } a>1 \text { and } n=\alpha_{k} q^{k} .\end{cases}
$$

In all cases we have that $\lambda \in \mathcal{B}_{n}(q, p)$. 
Proof. Let us first analyse the case where $\beta_{k}>0$. It is clear that $\lambda$ is well defined and that we have $C_{p}(\lambda)=(a)$ (since $\left.h_{2,1}(\lambda)=w p\right)$. Moreover we observe that $\left|\mathcal{H}_{1}^{q^{k}}(\lambda)\right|=\beta_{k}-1$, $\left|\mathcal{H}_{2}^{q^{k}}(\lambda)\right|=0$ and that $\sum_{j \geq 3}\left|\mathcal{H}_{j}^{q^{k}}(\lambda)\right| \leq \zeta_{k}$. It follows that $\left|\mathcal{H}^{q^{k}}(\lambda)\right| \leq \alpha_{k}-1$ and hence that $q$ divides $\chi^{\lambda}(1)$, by Lemma 3.2 .

We assume now that $\beta_{k}=0$ and let $\lambda=\left(a, 1^{w p}\right)$. Clearly $C_{p}(\lambda)=(a)$. Moreover, analysing again the hook-lengths in $[\lambda]$ and using Lemma 3.2 we deduce that $q$ divides $\chi^{\lambda}(1)$ unless $a=1$ or $n=\alpha_{k} q^{k}$. In these two cases we choose $\lambda$ as described in the statement and we verify that $C_{p}(\lambda)=(a)$ and that $q$ divides $\chi^{\lambda}(1)$, again using Lemma 3.2 .

Corollary 3.12. Let $a, p w$ and $\lambda$ be as in Lemma 3.11. Let $\phi$ be an irreducible constituent of $\left(\chi^{\lambda}\right)_{\mathfrak{A}_{n}}$. Then $\phi \in B_{0}\left(\mathfrak{A}_{n}\right)$ has degree divisible by $q$.

Proof. As usual, if $q \neq 2$ then the statement follows easily from Lemma 3.11. When $q=2$ the parameter $\beta_{k}$ is the coefficient of $2^{k}$ in the binary expansion of $a$. Since $a<p \leq w p<n$ we deduce that $\beta_{k}<\alpha_{k} \leq 1$, hence $\beta_{k}=0$. Moreover, as usual we only need to analyse the situations where $\lambda=\lambda^{\prime}$. It is easy to check that this never occurs. Hence $\chi^{\lambda}(1)=\phi(1)$ is even.

Proof of Theorem $C$. The statement of Theorem 3.5 concerning $\mathfrak{S}_{n}$ follows from Lemmas 3.7, 3.9 and 3.11, On the other hand, the part of Theorem 3.5 concerning $\mathfrak{A}_{n}$ follows from Corollaries 3.8, 3.10 and 3.12, As already remarked at the beginning of the section, Theorem 3.5 implies Theorem C.

In order to prove Theorem $\mathrm{C}$ without the assumption $q<p$, we would need a radically different combinatorial approach. This is not directly relevant for the purpose of the present article. Nevertheless, it could be material for further investigation.

\section{Simple groups of Lie type AND SPORADIC GROUPS}

The aim of this section is the proof of Theorem $\mathrm{B}$ on the principal blocks for the remaining non-abelian simple groups. The case of sporadic groups is immediately checked from the known character tables, leading to the unique counter-example $M_{22}$ at the prime 7 , so by the classification of the finite simple groups we are left to deal with the finite simple group of Lie type.

Theorem 4.1. Let $S$ be a finite simple group of Lie type and $\ell$ an odd prime dividing $|S|$. Then the principal $\ell$-block of $S$ contains an irreducible character of even degree.

Proof. We discuss the various cases. First assume that the defining characteristic $p$ of $S$ coincides with the (odd) prime $\ell$. By a well-known result then the only irreducible character of $S$ not contained in the principal $p$-block is the Steinberg character, whose degree is a power of $p$, hence in particular odd. The claim in this case now follows from the fact that a non-abelian simple group has order divisible by at least three distinct primes together with the remaining part of the proof. (Alternatively, a non-abelian simple group possesses an irreducible character of even degree by a result of Willems. By what we said before that character will lie in the principal $p$-block.)

We may hence assume that $\ell$ is not the defining characteristic of $S$. So $S$ is not a Suzuki group nor one of the big Ree groups. We now set up the following notation. Let $\mathbf{G}$ be 
a simple algebraic group of simply connected type over an algebraic closure of the finite field with $p$ elements, and $F: \mathbf{G} \rightarrow \mathbf{G}$ a Steinberg map, such that $S=G / Z(G)$, where $G:=\mathbf{G}^{F}$.

By [CE94, Thm.] the principal $\ell$-block of $G$ lies in the union of Lusztig series

$$
\mathcal{E}_{\ell}(G, 1)=\coprod_{t} \mathcal{E}(G, t)
$$

with $t$ running over the (semisimple) $\ell$-elements of the dual group $G^{*}$ modulo conjugation, and in particular, it contains the semisimple character(s) $\chi_{t}$ from each series $\mathcal{E}(G, t)$. Now the degree of $\chi_{t}$ is given by

$$
\chi_{t}(1)=\left|G^{*}: C_{G^{*}}(t)\right|_{p^{\prime}} .
$$

In particular this provides a character of even degree in the principal $\ell$-block of $G$ if $t$ does not centralise a Sylow 2-subgroup of $G^{*}$. This is an even degree character in the principal $\ell$-block of the simple group $S=G / Z(G)$ if it has $Z(G)$ in its kernel, which happens if and only if $t \in\left[G^{*}, G^{*}\right]$. Thus, to conclude we need to construct an $\ell$-element $t \in\left[G^{*}, G^{*}\right] \cong S$ not centralising a Sylow 2-subgroup of $G^{*}$.

First assume that $\ell$ does not divide the order of the centraliser of a Sylow 2-subgroup of $S$. Then clearly any non-trivial $\ell$-element of $S$ is as required. Now by [KM03, Thm. 7] a Sylow 2-subgroup $P$ of $S$ is self-centralising, unless $G$ is one of $\operatorname{SL}_{n}(q), \mathrm{SU}_{n}(q), E_{6}(q)_{\mathrm{sc}}$ or ${ }^{2} E_{6}(q)_{\mathrm{sc}}$. So it remains to consider these four families of groups. First assume that $G=\mathrm{SL}_{n}(q)$, and let $2^{t_{1}}+\cdots+2^{t_{r}}$, with $t_{1}<\ldots<t_{r}$ be the 2 -adic expansion of $n$. Then $P C_{S}(P) / P$ is a direct product of at most $r-1$ cyclic groups of order dividing $q-1$, so we may assume that $r \geq 2$ and $\ell \mid(q-1)$. Now let $t \in \mathrm{GL}_{n}(q)$ be an $\ell$-element with two distinct eigenvalues, one with multiplicity $2^{t_{r}}+1$, the other with $n-2^{t_{r}}+1$ (respectively one with multiplicity $2^{t_{r}}-1$, the other with multiplicity 2 if $r=2$ and $t_{1}=0$; respectively with three distinct eigenvalues when $n=3)$. Then the image of $t$ in $G^{*}=\operatorname{PGL}_{n}(q)$ does not centralise a Sylow 2-subgroup of $G^{*}$. If $G=\mathrm{SU}_{n}(q)$, then again $P C_{S}(P) / P$ is a direct product of at most $r-1$ cyclic groups, of order dividing $q+1$ this time, with $r$ as before. Hence in this case $\ell$ divides $q+1$, and we can construct an element $t$ as before.

For $S=E_{6}(q)$ the centraliser of a Sylow 2-subgroup contains a cyclic subgroup of order dividing $q-1$. Hence again $\ell$ divides $q-1$. Now take $t$ an $\ell$-element in the centre of a Levi subgroup $L$ of $S$ of type $A_{5}$. Then $L$ is the full centraliser of $t$ (as $L$ is a maximal reductive subgroup of $S$ ), but its index in $S$ is even, so $t$ is as required. Finally, for $S={ }^{2} E_{6}(q)$, we need to consider divisors $\ell$ of $q+1$, and here any $\ell$-element in the centre of a Levi subgroup of twisted type ${ }^{2} A_{5}$ will do. The proof is complete.

\section{REFERENCES}

[CE94] M. Cabanes, M. Enguehard, On unipotent blocks and their ordinary characters. Invent. Math. 117 (1994), 149-164.

[Is76] I. M. IsAACs, Character Theory of Finite Groups. Academic Press, New York-London, 1976.

[It51] N. Ito, Some studies of group characters. Nagoya Math. J. 2 (1951), 17-28.

[Ja79] G. D. JAmes, The Representation Theory of the Symmetric Groups. Lecture Notes in Mathematics, vol. 682, Springer, Berlin, 1978.

[JK81] G. JAmes, A. Kerber, The Representation Theory of the Symmetric Group, Encyclopedia of Mathematics and its Applications, vol. 16, Addison-Wesley Publishing Co., Reading, Mass., 1981. 
[KM03] A. S. Kondrat'Ev, V. D. Mazurov, 2-signalizers of finite simple groups. (Russian) Algebra Logika 42 (2003), 594-623, 638; translation in Algebra Logic 42 (2003), 333-348.

[Mac71] I. G. MaCDonaLD, On the degrees of the irreducible representations of symmetric groups. Bull. London Math. Soc. 3 (1971), 189-192.

[Mi86] G. Michler, A finite simple group of Lie-type has $p$-blocks with different defects, $p \neq 2$. J. Algebra 104 (1986), 220-230.

[Nav98] G. Navarro, Characters and Blocks of Finite Groups. London Mathematical Society Lecture Note Series, 250. Cambridge University Press, Cambridge, 1998.

[NRT17] G. Navarro, G. Robinson, P. H. Tiep, On real and rational characters in blocks. Int. Math. Res. Notices (2017), doi: 10.1093/imrn/rnx170.

[NW01] G. Navarro, T. Wolf, Character degrees and blocks of finite groups. J. reine angew. Math 531 (2001), 141-146.

[O194] J.B. Olsson, Combinatorics and Representations of Finite Groups. Vorlesungen aus dem Fachbereich Mathematik der Universität Essen, Heft 20, 1994.

Trinity Hall, University of Cambridge, Trinity lane, CB21TJ, UK

E-mail address: eg513@cam.ac.uk

FB Mathematik, TU Kaiserslautern, Postfach 3049, 67653 Kaiserslautern, Germany

E-mail address: malle@mathematik.uni-kl.de

ICMAT, Campus Cantoblanco UAM, C/ Nicolás Cabrera, 13-15, 28049 Madrid, Spain

E-mail address: carolina.vallejo@icmat.es 